
\title{
28 Research Square \\ Exploring StarD13 complex role in mediating the spread of lung cancer
}

\author{
Maria Al-Haddad \\ Rayane El-Rif \\ Samer Hanna \\ Leila Jaafar \\ Rayanne Dennaoui \\ Sandra Abdellatef \\ Veronika Miskolci \\ Dianne Cox \\ Louis Hodgson \\ Mirvat El-Sibai
}

\section{Video Byte}

Keywords: Cell Communication and Signaling, StarD13, Rho GTPases, invadopodia, migration, invasion, lung cancer, locomotion, adhesion, guanosine triphosphatases, motility, proliferation, adenocarcinoma, Steroidogenic acute regulatory protein-related lipid transfer domain-containing protein 13, DLC2, tumor suppression, siRNA, A549 cells, metastasis, FRET

Posted Date: November 12th, 2020

DOl: https://doi.org/10.21203/rs.3.rs-106667/v1

License: (c) (1) This work is licensed under a Creative Commons Attribution 4.0 International License. Read Full License 


\section{Abstract}

Lung cancer is the second most common form of cancer worldwide. The spread of lung cancer within the body is a complex process that hinges on tumor cells' ability to migrate. One family of proteins known as Rho GTPases is known to play a central role in regulating the cell mechanics required for migration. That role is more complex that initially thought and many cross talks are involved, as a recent study highlights for the GTPase StarD13. Initial experiments showed that StarD13 is a tumor suppressor in lung adenocarcinoma, confirming previously reported results. Interestingly, StarD13 is also required for the cancer cells to migrate. StarD13 regulates cell motility by modulating the activation of RhoA and Rac1 proteins downstream. Abolishing RhoA and Rac1 in lung cancer cells was shown to compromise cells' ability to migrate. What further proves the complexity of cancer metastasis, is that while abolishing StarD13 stopped cell migration, it actually enhanced cancer invasion in 3D. This study showed a novel role of StarD13 as a tumor suppressor by inhibiting Cdc42-mediated invadopodia, which are necessary for cancer invasion. Further exploring StarD13's complex role in cell migration and invasion could lead to more targeted therapies for patients with lung cancer. 